\title{
Special Issue of ADAM on Symmetries of Graphs and Networks - Call for Papers
}

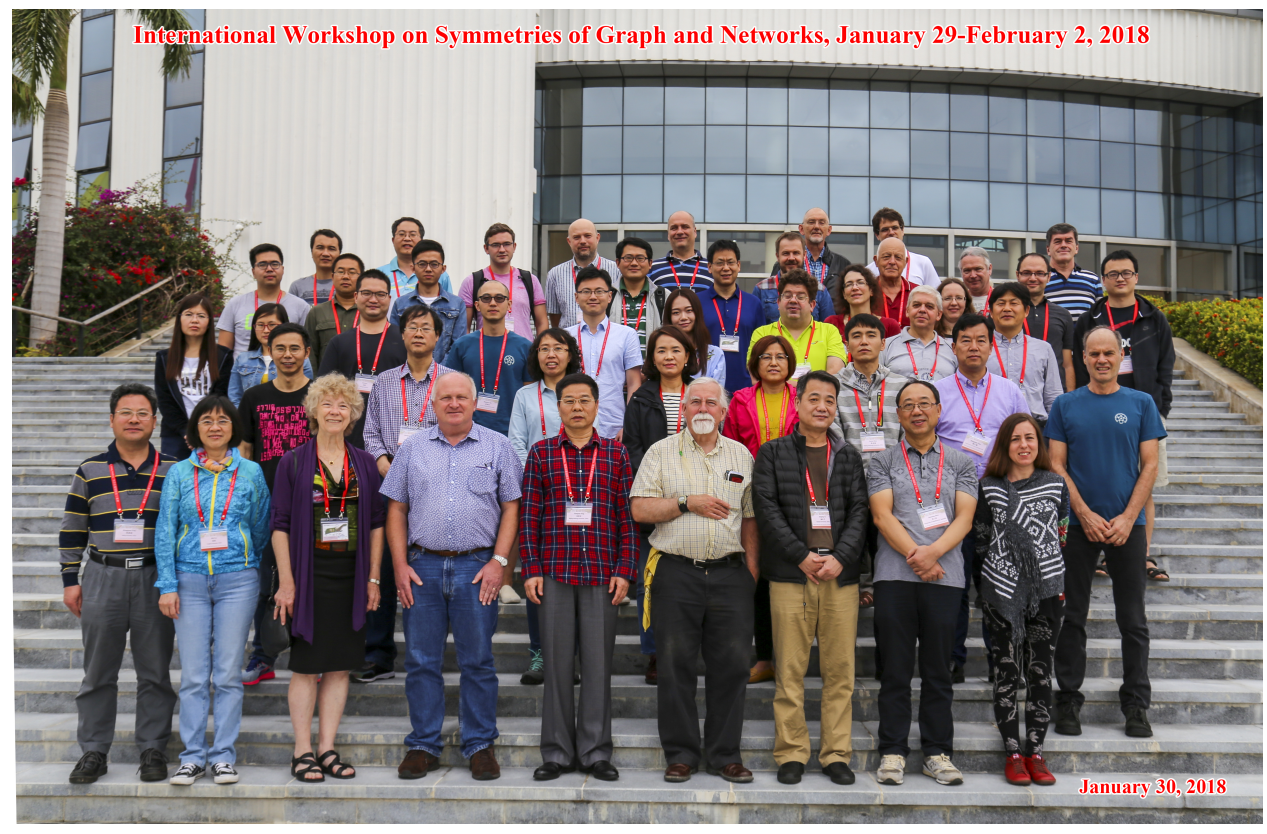

This is a call for submission of papers for a special issue of the journal The Art of Discrete and Applied Mathematics (ADAM), on topics presented or related to talks given at the TSIMF workshop on 'Symmetries of Graphs and Networks' held at Sanya (China) in January 2018. The Sanya workshop added to the series of conferences and workshops on symmetries of graphs and networks initiated at BIRS (Canada) in 2008 and progressed in Slovenia every two years from 2010 to 2016.

The Art of Discrete and Applied Mathematics (ADAM) is a modern, dynamic, platinum open access, electronic journal that publishes high-quality articles in contemporary discrete and applied mathematics (including pure and applied graph theory and combinatorics), with no costs to authors or readers. This special issue, however, will be also available in printed form for purchase.

Papers should be submitted by 31 December 2018, via the ADAM website https: / / adam-journal.eu/index.php/ADAM. A template and style file for submissions can be downloaded from that website, or obtained from one of the guest editors on request. The ideal length of papers is 5 to 15 pages, but longer or shorter papers will certainly be considered. Papers that are accepted will appear on-line soon after acceptance, and papers that are not processed in time for the special issue may still be accepted and published in a subsequent regular issue of ADAM.

Marston Conder and Yan-Quan Feng Guest Editors 